\title{
Europa Lander Mission: A Challenge to Find Traces of Alien Life
}

\author{
Lev Zelenyi ${ }^{1}$, Oleg Korablev ${ }^{2}$, Elena Vorobyova ${ }^{2,1}$, Maxim Martynov ${ }^{3}$, \\ Efraim L. Akim ${ }^{4}$, Alexander Zakahrov ${ }^{1}$ \\ ${ }^{1}$ Space Research Institute (IKI), Moscow, Russia, Fax: +7 4953331248 \\ ${ }^{2}$ IKI \\ ${ }^{3}$ Lavochkin Assosiation, Russia \\ ${ }^{4}$ Keldysh Institute of Applied Mathematics, Russia \\ email: korab@iki.rssi.ru
}

\begin{abstract}
An international effort dedicated to science exploration of Jupiter system planned by ESA and NASA in the beginning of next decade includes in-depth science investigation of Europa. In parallel to EJSM (Europa-Jupiter System Mission) Russian Space Agency and the academy of Science plan Laplace-Europa Lander mission, which will include the small telecommunication and science orbiter and the surface element: Europa Lander. In-situ methods on the lander provide the only direct possibility to assess environmental conditions, and to perform the search for signatures of life. A critical advantage of such in situ analysis is the possibility to enhance concentration and detection limits and to provide ground truth for orbital measurements. The science mission of the lander is biological, geophysical, chemical, and environmental characterizations of the Europa surface. Remote investigations from the orbit around Europa would not be sufficient to address fully the astrobiology, geodesy, and geology goals. The science objectives of the planned mission, the synergy between the Europa Lander and EJSM mission elements, and a brief description of the Laplace-Europa Lander mission are presented.
\end{abstract}

Keywords. Europa; Jupiter; Galilean satellites; habitability; space research.

\section{Introduction}

The investigation of giant planets and their satellites is important for understanding of the formation and evolution of our Solar system, measuring distribution of chemical elements, study of internal structure and atmospheres of giant planets and their satellites, assessing the habitability of these distant worlds. It has been an important part of NASA research program starting from Pioneer and Voyager missions in the beginning of $1970^{t h}$. Jupiter system arouses the most interest of the scientific community after pioneering Voyager and Galileo missions. Along with the planet and its extraordinary magnetosphere, the Galilean satellites of Jupiter, and Europa in particular, attract special attention.

To some extent Europa is a similar to the Moon. The radius of Europa is a little smaller, but the average density (about $3 \mathrm{~g} \mathrm{~cm}^{-3}$ ) is close to that of the Moon $\left(3.3 \mathrm{~g} \mathrm{~cm}^{-3}\right)$, suggesting that Europa is mostly a mineral body. Like the Moon, Europa and other Galilean satellites are nearly in synchronous rotation when the rotation period almost equals the orbital period and the satellite faces the planet with always the same side.

Water ice on the surface of Europa has been discovered by means of ground-based astronomy long before Voyager spacecrafts arrived to Jupiter (Kuiper, 1957, Moroz, 1965, Johnson and McCord 1971, Pilcher et al., 1972). Near-infrared spectra of Europa and Ganimede demonstrate a broad feature near $1.5 \mu \mathrm{m}$ associated with $\mathrm{H}_{2} \mathrm{O}$ ice band. The 
dayside surface temperature was estimated at 120-135K. First images of Europa's surface have been obtained in 1979 during Voyager 1 and 2 flybys. The most part of surface looks relatively smooth and bright; few equatorial areas being darker and spotted. The observed density of impact craters is far lower than on other Solar system bodies. The main morphological feature of Europa surface is a network of dark linear details, extending over thousands of kilometers. The smoothness of the surface indicates that the ice remained relatively warm, soft and mobile during an important part of the geological history of the satellite. Many observations indicated that there might be a global liquid ocean beneath the icy crust, which could be theoretically a habitat for some form of life (Reynolds et al., 1983).

In 1995 the Galileo spacecraft entered into the orbit of Jupiter's satellite. Since 1997 the mission was focused on Europa. High resolution (up to several meters) images of Europa have been obtained (Greeley et al., 1998a). The width of many linear features appears to be only about one kilometer. It was proposed that the prominent linear, often double and multiple ridges have been formed as a result of extrusion of viscous material. The Earth analog of such process could be the formation of middle oceanic ridges as a result of repeated magma flows. The main source of energy in the tectonic process on Europa is a tidal interaction with Jupiter and other satellites. The resonance keeps the satellite from perfectly circular orbit, inducing a forced eccentricity that results in tidal flexing and internal dissipation of energy. The formation of the bands and lineations of the shell could be explained by global stress fields due orbital eccentricity, orbital recession, and nonsynchronous rotation. The displacement of the tidal bulge due to nonsynchronous rotation is the best explanation of ridges and cycloidal features assuming relatively thin ice crust (McEwen 1986, Greeley et al., 1998b, Hoppa et al., 1999).

Impact craters rates have shown the age of most areas does not exceed 100 million years (Zahnle et al., 2003, 2008). The young surface requires recent geologic activity, which is more likely in the presence of liquid water. The appearance of chaotic terrains, rafts, and many other geological evidences presume recent release of water on the surface. However, a search for on-going geologic activity on Europa surface over the 20-year period between Voyage and Galileo observations has shown no detectable changes (Phillips et al., 2000).

Gravity measurements by the Galileo show that Europa is an internally differentiated rocky body with the thickness of the outer water shell in the range of about 80 to 170 kilometers (Anderson et al., 1998). These data cannot distinguish between water in liquid and ice phases. Current models of the internal structure of differentiated Europa predict a Fe-FeS core with radius of order 500-600 km depending on model, mineral mantle about $1000 \mathrm{~km}$ and water crust below $200 \mathrm{~km}(80-160 \mathrm{~km})$ (Kuskov and Kronrod, $2005)$. The contact of the putative liquid ocean and the mineral floor is a key point for a hypothesized habitat of the Europan ocean.

The exploration of outer planets continues to play an important role in the program of leading space agencies. Currently JUNO, a New Frontiers mission to study the atmosphere, magnetosphere and gravitational field of Jupiter is being prepared by NASA for launch in August 2011 (Matousek 2007). After analyzing several concepts of Europa missions of different cost/complexity levels since 1996 (Langmaier 2008; Gershman, 1999, Gershman, 2003), NASA concluded that multiple and single flyby missions have significantly less science value when compared to a dedicated moon orbiter. A first dedicated mission at Europa consisting of a single lander was considered risky and limited to only one site. Participating in the successful Cassini-Huygens programme ESA actively joined the program of outer planets exploration. In 2007 two prominent 
developments, ESA Cosmic Vision Class L proposal and NASA Flagship mission proposal were merged into a joint space mission to the Jupiter system: Europa Jupiter System Mission (EJSM, http://opfm.jpl.nasa.gov/europajupitersystemmissionejsm/) with an approximate launch date in 2020-2022 (Blanc et al., 2009, Clark et al., 2009). Recently this project was selected among two outer planet missions for further development (Phase A) with final selection planned for 2012-2013. The project includes two spacecrafts for exploration of Jupiter, Ganimede and Europa. In the framework of EJSM, NASA plans Jupiter Europa Orbiter (JEO) for investigation Jupiter, Io and focused on Europa and its habitability. It is designed to follow-up on the major discoveries of the Galileo and Voyager missions at Europa, and in particular to characterize its ocean. JEO will be built to withstand the intense radiation at Europa orbit, and would consist of an orbiter with 11 science instruments designed for extensive mapping of Europa. On the way to Europa, JEO will tour the Jovian system and make routine and frequent observations of Jupiter, its satellites and its environment. ESA plans Jupiter Ganymede Orbiter (JGO) for investigation of Jupiter, Ganimede and Callisto. It would consist of a solar powered orbiter with 10 science instruments designed for remote sensing of Jupiter and the inner satellites and for extensive mapping of Ganymede. Prior to Ganymede arrival, JGO will intensely investigate Callisto from a resonant orbit, and make extensive observations of the Jupiter system to complement those of JEO. The Jupiter Magnetospheric Orbiter (JMO) proposed by the Japanese Aerospace Exploration Agency (JAXA) may be included into EJSM project to characterize plasma environment of the Jupiter system. JMO would undertake detailed in-situ studies of Jupiter's magnetosphere affording the opportunity for "3-point" investigations of the Jupiter system together with JGO and JEO.

Traditionally the Soviet, and later the Russian space program was limited to the inner part of Solar system (Moon, Mars and the most successfully Venus). Still, some preliminary studies of scientific projects to Jupiter system were carried out in the USSR in the $80^{\text {th }}$. Starting from 2007 Russian Academy of Sciences and Roscosmos considers the possibility to include in EJSM a Europa surface element, a soft lander, independently launched by Russia [Blanc et al., 2009]. The scientific objectives of this lander will be to search for signatures of possible present and extinct life, and in situ studies of Europa internal structure, surface and environment. After preliminary assessment of the project in 2008-2009 it has been decided to complement the mission with an orbiter of Europa, to support independent data relay. The Orbiter observations will be also used for the selection of the landing site in real time, and conduct supportive science experiments. This mission nominated Laplace - Europa Lander will continue with assessment study in 2010-2012.

Cooperating with the EJSM mission, the Russian Europa Lander will: 1) complement and support remote sensing by JEO and JGO investigations with in-situ measurements to provide ground truth for the remote sensing data; 2) complement and validate remote sensing data by JEO and JGO in the Jupiter system by remote observations from Russian Europa Orbiter with different instruments; 3) provide a multipoint sounding of Jupiter plasma environment together with JGO, JEO and possibly with Japanese JMO. In turn, Europa Lander will benefit from high-resolution mapping and other data collected by JEO for landing site selection. Also, the possibility of data relay from Europa Lander via JGO is being considered. The launch of Europa Lander should follow the launch of JEO, and the mapping information from JEO should arrive during the cruise/approach phase of Europa Lander mission. 


\section{Europan Ocean}

The existence of a global subsurface ocean on Europa is supported by many above observations. Already the analysis of Voyager imaging suggested that orientations of the linear features cannot be satisfactorily explained by stress fields due to the nonsynchronous rotation. In the meanwhile, stress directions inferred for many bands, are consistent with stresses resulting from sliding of the crust of Europa as a single unit (Schenk and McKinnon 1989). Such a reorientation, denominated as polar wander, may result from spatial variations in the thickness of the shell (Ojakangas and Stevenson 1989). Such polar wander probably also explains symmetry patterns in the distribution of chaotic terrain, pits, and uplift features (Greenberg et al., 2003; Schenck et al., 2008].

The polar wander is a good evidence of a global ocean in the past. The most convincing evidence of the contemporary global ocean comes from Galileo magnetic field measurements (Kivelson et al., 2000; Zimmer et al., 2000). The induced magnetic field of Europa variable in direction and strength in function of Europa position within Jupiter's strong magnetic field was predicted by Colburn and Reynolds (1985). The data cannot be explained by localized pockets of salty water, and require a nearly complete spherical shell of salty water, with likely low salinity, lower than that of the Earth Ocean (Schilling et al., 2004; Hand and Chyba 2007). The best estimate of the ice thickness from conductivity models is $4 \mathrm{~km}$.

Estimates of Europa's ice-shell thickness range from several hundred meters to $\geqslant 30 \mathrm{~km}$. All of them are model dependent, and suffer from unknown parameters and insufficiently precise observations. Two major lines of evidence based on tidal deformations due to orbit eccentricity and asynchronous rotation, see Billings and Kattenhorn (2005) for a review. Estimates based on tidal flexing (e.g., Williams and Greeley, 1998, Billings and Kattenhorn 2005) give a thin shell of brittle ice, under which many authors suppose a thick convective low-viscosity layer. Buoyancy models (e.g., Carr et al., 1998, Williams and Greeley, 1998) basing on somewhat limited stereo and photoclinometric topography of the satellite, and estimation based on the resistance of the ice shell to tidal stress leading to formation of cycloids (Hoppa et al., 1999; Greenberg et al., 2000) indicate the total depth of the ice of about few kms. Crater depth and morphology analysis (Greeley et al., 1998b; Schenk, 2002] provide estimates of the ice shell varying from few to 18-20 $\mathrm{km}$. The extreme position in this debate, a thick shell of a few tens of kilometers likely convective in its lower part (e.g., Pappalardo et al., 1999) is supported by thermodynamic models (Hussmann et al., 2002; Tobie et al., 2003). The uncertainty in thickness translates directly into an uncertainty in the heat transfer mechanism: if the shell is thick, the rigid surface could be underlain by a layer of convecting water ice (e.g., Tobie et al., 2003; Showman and Han, 2004), whereas a thin shell would instead transport the heat by conduction, and can account for melting of water through the surface (Greenberg et al., 1999).

In spite of numerous observational evidences the existence of the global ocean on Europa remains a subject of debate (Carr et al., 1998; Pappalardo et al., 1999; Stevenson 2000; Schenk et al., 2008). Galileo observations and measurements cannot give a definitive constraint about the thickness of the ice shell, provided the ocean is there. The icy crust is likely variable in thickness, inhomogeneous in composition, and possibly evolving in time on geologically short time scales (Zahnle et al., 2003; Figueredo and Greeley 2004). Likely thin or locally thinned ice shell allows ocean water to reach the surface, possibly periodically on a relatively short time scales.

The thickness of the shell above the ocean is of fundamental importance both for the habitability of the ocean (because it is a quantitative indication of energy available from 
different sources), and for the ability to discover a signature of life on the surface or shallow subsurface of Europa.

\section{Habitability of Europa: Alien Life?}

If the subsurface ocean exists on Europa, it is characterized by supply of biologically important elements from the floor, and energy sources, from tidal effects, radioactivity, and geochemical mechanisms. Europan subsurface ocean environment may be similar to that of the deep ocean hydrothermal vents on Earth where remarkable life forms have been detected. There is a fundamental difference among finding life at the depth on the Earth (with biomass may be comparable to that on the surface, see e.g., Whitman et al., 1998) or even the finding the life on Mars and the possibility to find life on Europa. The existence of "deep" life on Earth is not a proof that life has originated at depth. Similarly, interplanetary transfer of microorganisms could be a possible way of insemination of Mars or other bodies in the solar system. Situation is very different on Europa. According (Pierazzo and Chyba, 2001) about $10^{12} \mathrm{~kg}$ of carbon ( $\sim 0.1 \%$ of the Earth biomass) could accumulate on Europa since its formation. However, there is no atmosphere on Europa, and interplanetary dust particles or small meteorites hit the surface at very high velocities leading to their total destruction. Therefore, the mechanism, which might deliver the prebiotic organics to early Earth (Mukhin et al., 1989; Chyba and Sagan, 1992), or possibly inseminated Mars (Mileikowsky et al., 2000), would hardly work on Europa.

It means that if life is found on Europa, it is (1) life originated in the depth of subsurface ocean independently of the free energy from the Sun, and (2) life originated independently of the life on the Earth, likely, an alien or endemic biology. Such a discovery would mean that the traditional view of planetary habitability, widely discussed in relation with exasolar planets, and based on distance to the star and the surface temperatures should be broadened (Chyba and Philips, 2002).

The analysis of Galileo mission data enables to develop new astrobiological concepts that speculate upon extant life on Europa (Reynolds et al., 1983; Sieger et al., 1998; Kargel et al., 2000; Chyba 2000). Several basic features of Europan evolution has been postulated by Kargel et al. (2000): (1) initial carbonaceous chondrite rock; (2) global primordial low-temperature aqueous differentiation and formation of an impure primordial hydrous crust; (3) brine evolution and intracrustal differentiation; (4) degassing of Europa's mantle and gas venting; (5) hydrothermal processes, and (6) chemical alteration of the surface. These assumptions would result in a more realistic ocean/asthenosphere/ lithosphere model and in crust/ocean enriched by magnesium and sodium sulfates, substances discovered on the surface of Europa by Galileo spectroscopy. A wide range of possible ocean/crust compositions is the reason to construct different scenarios for the origin and evolution of putative ecosystems. The main energy sources, which could provide the support for the extant life at Europa are the tidal heating and the convection (Reynolds et al., 1983), radiolytic generation of oxidants (Chyba 2000; Sieger et al., 1998), chemoautotrophy (Kargel et al., 2000; Sieger et al., 1998), and possibly ionotrophy (Irwin and Schulze-Makuch, 2003). Microbial metabolites, dead biomass and also primordial organics must serve as a nutrient and energy sources for the attendant heterotrophic communities. From these considerations one may develop models of complicated and diverse ecosystems, protected from harsh surface conditions, and evolving successfully in the planetary interior. These potential habitats are based on aquatic biochemistry in salty ocean, ice, and on the seafloor. 
Although the conditions at Europa and at the Earth differ significantly, extreme terrestrial ecosystems have been always considered and extensively analysed as useful models for astrobiology. There are two basic reasons for these considerations:

First, terrestrial ecosystems serve as (the only to date) basis for definition of life. Commonly, the Earth-centric view point implies the Earth-centric perspective for spreading of biological life. But the utimate goal of astrobiological search for life is to find an isolated extraterrestrial biosphere. One should agree with Conrad and Nealson (2001) who have claimed that we must define life in universal measurable terms and should have a clear idea of the end-member states for this search - what does life, or the absence of life look like at multiple spatial and temporal scales. The chemical composition, structures, energy consumption etc., have been proposed as examples of such universal characteristics of life. The totality of above characteristics has to be analysed in order to choose appropriate criteria, testifying life in putative Europan ecosystems.

Second, the considerable optimism regarding the habitability of Europa is to large extent based on the presence of strong tolerance of microbial and biochemical activity in Earth environments, which could be evaluated as possible Europan analogues. Prebiotic processes under hydrothermal conditions may have been important in the origin of terrestrial life (e.g., Mukhin, 1976; Cody et al., 2000). There has been a lot of research on the ability of microorganisms to grow in extreme environments with respect to temperature, salinity, acidity, radiation, and pressure (Kargel et al., 2000; Marion et al., 2003). Polar investigations confirm that cold environments served as a refuge for life during long-term glaciations. Cold ecotopes are biochemically active at subzero temperatures, and represent a number of features characteristic for adaptation of living microbial populations to low temperatures (Abyzov, 1993; Vorobyova et al., 1996, 1997; Priscu et al., 1999; Priscu, 2002; Castello and Rogers, 2005; Gilichinsky et al., 2007). In all cases active life at subzero temperatures occurs in presence of liquid water, maintained due to highly concentrated brines, or at water-mineral interface where the freezing temperature increases.

Several extreme ecosystems on the Earth have been proposed as potential Europa analogs for biomarkers' investigation, selection of life criteria, and validation of experimental approaches. These include lake and sea-ice biotopes, deep-ice cores, permafrost, and deep sea hydrothermal vents. The most intriguing ones are the lake Vostok in Antarctica, and chemosynthetic communities in the Gulf of Mexico at 540-m depth (Sassen et al., 1999). In the Golf of Mexico oxidation of methane hydrates drives biochemical processes which support complex chemosynthetic communities and consumers as well. It is supposed that similar hydrothermal vents exist below the most recently resurfaced areas of Europa ice crust. These sites are the most promising for the search of extant life.

The particular feature of a putative Europan or other Jovian satellites' biospheres in comparision with Mars is that they could not be completely dormant (anabiotic). In contrast to Mars, Jovian satellites seem not suffered great cataclysms during their evolution, environmental conditions have not change drastically, and putative life developed through specific adaptations. If biosphere has emerged on Europa it should be active now.

Whether the life could have originated in the absence of sunlight? If so, the scientific rewards for successful search for life on Europa could hardly be greater. The Lander provide a unique possibility of finding traces of biotic or pre-biotic materials from the recent ocean outflows on the surface, which explains high science merit of such mission. 


\section{Science Goals of Europa Lander Mission and Possible Measurement Strategies}

Scientific questions to be addressed by instruments on the surface of Europa and even by deep submarine expeditions were extensively discussed in the refereed literature (Chyba and Philips, 2002; Cooper et al., 2002; Gershman et al., 2003; Zimmerman et al., 2005; Langmaier and Elliott, 2008 and the references therein). We repeat the most obvious science goals, effectively addressed by surface science.

The main scientific questions of Europa exploration can be formulated as follows:

- Is there a liquid water beneath the ice crust of Europa?

- Does the global ocean really exist on Europa?

- What is the depth and distribution of the icy crust?

- Are environmental conditions on Europa suitable for life?

- Are there traces of extinct life on Europa?

- Are there evidences of life on Europa at present time?

Clearly, remote studies from the orbit around Europa would not be sufficient to fully characterize surface environment and to address astrobiology goals. Measurements on the surface are important for geodesy and geology, to study the Europa ocean and to characterize locally the ice crust. Laplace-Europa Lander project will tackle the fundamental questions of internal structure, surface environment, and habitability of Europa. The main objectives of the mission will be to softly land on the chosen location, to collect multiple samples from one or multiple nearby sites to provide access to the shallow subsurface (to reach unaltered by radiation material). The landing site will be selected using the most accurate remote imagery to land on geologically youngest area, and to probe the material as recently exposed to the surface as possible. The following science and measurement objectives are being considered:

- To corroborate the theory of the liquid ocean, to characterize of the thickness and stiffness of the icy crust, to study the internal structure by means of different geophysical measurements; to characterize the seismicity of Europa, and to measure the magnetic field on the surface

- To conduct a detailed study of surface material, characterize physical (electrical and heat conductivity, stiffness, etc.) and chemical ( $\mathrm{pH}$, redox potential) parameters, analyze the composition of ice and admixtures, including isotopic ratios by means of gas chromatography with mass spectrometry (GCMS) and other methods;

- To characterize environment with particular attention to its capability to support life, and to search the traces of extinct or extant life in the surface and shallow subsurface (organic components, anions, cations, salinity, elements relevant to primary biological productivity, e.g. $\mathrm{N}, \mathrm{O}, \mathrm{P}, \mathrm{S}, \mathrm{Mg}$, potential metabolism products), isotopic composition $\left({ }^{13} \mathrm{C} /{ }^{12} \mathrm{C},{ }^{15} \mathrm{~N} /{ }^{14} \mathrm{~N}\right.$, etc.) at high sensitivity, by means of GSMS, Raman spectroscopy, other methods;

- To conduct observations and measurements in regional, local and micro scales, to study morphology and mineralogy of the surface and to validate remote orbital observations;

- To perform local measurements of radiation conditions, secondary ions, exosphere of the satellite and volatiles near the surface $\left(\mathrm{CH}_{4}, \mathrm{NH}_{3}, \mathrm{CO}_{2}\right.$, etc.)

A list of potential experiments to be considered on the surface of Europa, and their relevance to three major classes of science goals: Conditions, Composition, and Habitability is presented in Table 1 . This list comprises many experiments with duplicated science goals, and should not be considered as a model payload, but rather as a long list of potential candidates to the model payload. Most of these methods contribute to the 
Table 1. A list of possible instruments on the surface of Europa from a lander (adapted from Korablev et al., 2010).

\begin{tabular}{|c|c|c|c|c|c|}
\hline Instrument & Conditions & Composition & Habitability & Prototype & $\begin{array}{l}\text { Mass } \\
\text { (estimated) }\end{array}$ \\
\hline Seismometer & $\bullet$ & & $\circ$ & OPTIMISM/Mars 96 & $\begin{array}{l}495 \mathrm{~g}+\text { elec- } \\
\text { tronics }\end{array}$ \\
\hline Gravimeter & $\bullet$ & & ○ & GRAS/Phobos 11 & $250 \mathrm{~g}$ \\
\hline Tiltometer & $\bullet$ & & ○ & Huygens & $(300 \mathrm{~g})$ \\
\hline Magnetometer & $\bullet$ & $\circ$ & $\circ$ & MMO Bepi Colombo & $770 \mathrm{~g}$ \\
\hline TV camera set & $\bullet$ & $\circ$ & $\circ$ & $\begin{array}{l}\text { CIVA/Rosetta; Phobos } \\
11\end{array}$ & $1200 \mathrm{~g}$ \\
\hline Optical microscope & $\bullet$ & $\bullet$ & $\circ$ & Beagle-2; Phobos 11 & $300 \mathrm{~g}$ \\
\hline IR spectroscopy & $\bullet$ & $\bullet$ & $\circ$ & $\begin{array}{l}\text { No direct prototype; tech- } \\
\text { nique well established }\end{array}$ & $(2000 \mathrm{~g})$ \\
\hline $\begin{array}{l}\text { IR close-up spec- } \\
\text { trometer }\end{array}$ & $\bullet$ & $\bullet$ & $\bullet$ & $\begin{array}{l}\text { CIVA/Rosetta } \\
\text { MicrOmega/ExoMars }\end{array}$ & $(1000 \mathrm{~g})$ \\
\hline GCMS & ० & $\bullet$ & $\bullet$ & $\begin{array}{l}\text { GAP/Phobos } 11 \\
\text { COSAC/Rosetta }\end{array}$ & $(5000 \mathrm{~g})$ \\
\hline Wet chemistry set & & $\bullet$ & $\bullet$ & Urey/ExoMars ${ }^{1}$ & $2000 \mathrm{~g}$ \\
\hline Immuno-arrays & & o & $\bullet$ & SOLID /ExoMars ${ }^{1}$ & $(1000 \mathrm{~g})$ \\
\hline ATR spectroscopy & $\circ$ & $\bullet$ & $\bullet$ & $\begin{array}{l}\text { MIMA/ExoMars }{ }^{1} \\
\text { FTS analyzer }\end{array}$ & $(2000 \mathrm{~g})$ \\
\hline $\begin{array}{l}\text { Raman } \\
\text { spectroscopy }\end{array}$ & $\circ$ & $\bullet$ & $\bullet$ & 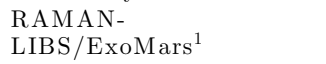 & $1100 \mathrm{~g}^{2}$ \\
\hline LIBS & $\circ$ & $\bullet$ & $\circ$ & $\begin{array}{l}\text { RAMAN- } \\
\text { LIBS } / \text { ExoMars }^{1}\end{array}$ & $1100 \mathrm{~g}^{2}$ \\
\hline Laser-ablation MS & $\circ$ & $\bullet$ & $\circ$ & LASMA/Phobos 11 & $1000 \mathrm{~g}$ \\
\hline $\mathrm{XRF}$ & $\circ$ & $\bullet$ & ○ & APXS/Rosetta & $640 \mathrm{~g}$ \\
\hline XRS & $\circ$ & $\bullet$ & $\bullet$ & No prototype & $(2000 \mathrm{~g})$ \\
\hline $\mathrm{XRD}^{3}$ & $\bullet$ & ○ & & XRD/ExoMars & $1200 \mathrm{~g}$ \\
\hline Various sensors & $\bullet$ & ○ & ○ & MUPUS/Rosetta & $2350 \mathrm{~g}$ \\
\hline Radiation dose & $\bullet$ & & $\circ$ & RADOM/Chandrayaan-1 & $100 \mathrm{~g}$ \\
\hline
\end{tabular}

Notes:

$\bullet=$ direct support; o =indirect support

${ }^{1}$ Experiments considered for ExoMars, but presently excluded from science package

${ }^{2}$ Target mass for both Raman and LIBS

${ }^{3}$ XRD experiment makes sense with a sample of pristine ice material from depth, non-altered, or little altered by radiation and meteorites.

assessment of the habitability of Europa, and many chemical analysis experiments have a high potential for biochemical detection of life (see Table 1). Specific life-contrasting tests might include isotopic ratios (GCMS and TDLAS, but likely concentration needed); chirality (difficult detection by UV methods, Raman, could also be assessed by GCMS); wet chemistry set, and immuno-arrays. A strategy to assess the feasibility of these experiments and a proper balancing between instruments proposed for a direct search of life and instruments for standard/advanced in-situ chemical and physical characterization of Europa is to be developed.

The most trustworthy experiments to be put on the surface of Europa identified so far are:

- seismometer, to estimate the thickness of the ice,

- a set of sensors for physical characterization

- chemical analytic package with high exobiology potential, based on GCMS

- IR spectrometer to link orbital and surface measurements

- a set of cameras, and microscopes

Three method to sample the surface, which are potentially compatible with the resources allocation of the lander are: the robotic arm/grinder, a drill to reach the depth of several tens of $\mathrm{cm}$ and to deliver the sample into the lander, and a melting probe with a mass of $\sim 5 \mathrm{~kg}$ (1 kg instrument) to reach the depth of $\sim 3$ meters. 
The science goals of the orbital element of the Laplace-Europa Lander mission are still to be considered. It is reasonable to duplicate some key investigations of NASA JEO, e.g., high-resolution imaging for landing site selection, lidar to characterize the figure of the satellite, near-IR mapping spectroscopy for surface composition, possibly a long-wave penetrating radar to map the thickness of the ice crust. A number of complementary measurements will be considered, to characterize in situ the ion and neutral composition of Europa environment, to measure the radiation dose, and to perform remote studies of Jupiter and other satellites.

\section{Mission concept}

A number of missions to explore Europa have been studied during the past decade. A comparison of mission architectures is presented in NASA report by Langmaier and Elliot (2008). This study has concluded that the most of the science at lowest mission risk can be achieved with a powerful science Europa orbiter. Such a Flagship-class mission, Europa Explorer (Clark et al., 2007) has become the basis of NASA share of the EJSM. However, previous studies of large Jupiter system missions included a number of surface elements (Greeley and Johnson, 2004).

An analysis of mass allocation for various landing strategies shows (Balint, 2004) that a classical soft lander is the most advantageous in terms of mass. A number of hard penetrator solutions are being considered for Europa (Gowen et al., 2010). However, the high-velocity penetrator concept for an airless body is yet to be demonstrated. Russian Laplace-Europa Lander mission includes a soft lander (total mass of $1210 \mathrm{~kg}$ ), and a small telecommunication and science orbiter $(395 \mathrm{~kg}$ ) (Zelenyi et al., 2010a). Comparing with many previous landers with mass below $\sim 400 \mathrm{~kg}$, Laplace-Europa Lander is relatively large and may afford a considerable science package.

Two variants of the interplanetary cruise are possible. First, a conventional scheme, including chemical propulsion and a series of gravitational maneuvers around Venus and the Earth has been considered. Alternatively, electric propulsion during the heliocentric cruise coupled with a single gravitational maneuver near the Earth can be employed. In either case the mass of filled transport modules (propulsion system) for such class of mission takes up to $80-85 \%$ from total mass of the spacecraft.

To enter into the orbit around Europa in the vicinity of Jupiter a series of gravitational maneuvers near Galilean satellites is required in order to save propellant mass. Extremely strong radiating belts of Jupiter have to be taken into account. Lengthy approach to the planet is unacceptable due to enormous cumulated radiation dose, destroying the subsystems of the spacecraft. The trajectory in the vicinity of Jupiter should be chosen in order to minimize the stay within Europan orbit, and to exclude whenever possible entering within the Io orbit. Estimations of charged particle fluxes and radiation doses under various shielding in different parts of the trajectory were made using different empirical models at each stage of the computations (Podzolko et al., 2009). The chosen sequence of gravitational maneuvers is shorter than two years. This stage is completed with the insertion into a circular polar orbit around Europa.

The calculations have shown, that even a heavy-class launch vehicle Proton with upper stage booster Breeze-M does not allow sufficient mass for both Orbiter and Lander to be delivered to Europa using chemical propulsion, even using multiple gravitational maneuvers. It is feasible, however when using the electric propulsion during the interplanetary cruise associated with gravitational maneuver near the Earth. In either case, a heavy-class launch vehicle is required to carry out the spacecraft (SC) to the escape trajectory. 
This scenario, using the electric propulsion and one gravitational maneuver near the Earth is accepted as the basis for the further analysis. The ballistic scheme of mission consists of following basic stages:

- Transfer to low earth orbit $(200 \mathrm{~km})$ by Proton launcher

- Acceleration to interplanetary trajectory by upper stage Breeze; jettisoning of Breeze;

- Earth-Earth cruise using electric propulsion;

- Gravitational maneuver at the Earth;

- Earth-Jupiter cruise using electric propulsion; jettisoning of electric propulsion module;

- Breaking in the sphere of Jupiter attraction and insertion into initial high-apogee orbit;

- Increasing the pericenter altitude to Ganymede orbit;

- Multiple rendezvous with Ganymede and Callisto to reduce the relative speed of approach to Europa;

- Insertion into a circular orbit around Europa with an altitude of $100 \mathrm{~km}$; jettisoning of the braking propulsion unit;

- Orbital flight; separation of the landing module;

- Deceleration of the landing module, and landing.

Once the spacecraft arrives to Jupiter system, it approaches Jupiter $\left(10^{5} \mathrm{~km}\right)$ and enters into an initial orbit. During the first approach the orbit is inclined at $40^{\circ}$ with respect to the equator plane to minimize harmful influence of radiation belts of Jupiter. This inclination is compensated by an impulse in apocenter, and the spacecraft enters in a transfer orbit with a pericenter of $9 \times 10^{5} \mathrm{~km}$, and an apocenter of $2 \times 10^{7} \mathrm{~km}$ in the plane of Galilean satellites. During further flight at resonance orbit a series of rendezvous with the satellites is conducted, allowing to reduce propellant required for the insertion into the orbit round Europa. This stage will last $\sim 23$ months (see Figure 1).

From this orbit remote studies of the surface will be conducted, and the landing site meeting certain topography conditions will be chosen. The landing module will be separated to perform an active soft landing onto the surface. The orbital module remains on the orbit and serves as a relay for the lander.

The landing is performed in two stages. First, the velocity of the landing module is reduced, than measurements of the altitude are performed, and the inertial speed of descent is defined. Second, the lander is rendered along the local vertical, and the vertical speed is reduced from about $3 \mathrm{~km} / \mathrm{s}$ to nearly zero at about $20 \mathrm{~m}$ above the surface.

\section{Mission design}

The mass breakdown of the Europa mission is given in Table 2. The spacecraft (see Figure 1) consists of four following basic elements:

- The electric propulsion transport module;

- The braking module.

- The orbiter;

- The lander.

The electrorocket transport module provides acceleration and deceleration during the heliocentric part of the flight. It is also responsible for the attitude control and stabilization of the spacecraft during this period. The traction is provided by means of eight plasma engines SPD-140 with a thrust of $\sim 0.17 \mathrm{~N}$ each, and a specific impulse of 28000 $\mathrm{m} / \mathrm{s}$. The xenon is used as the propellant. The engines are integrated in four blocks, each of them includes two SPD-140 engines and a 1-axis deployment/attitude control mechanism. The velocity vector is controlled aligning simultaneously the orientation of the four 
engine blocks, and the solar panels. Measures are taken to prevent contamination of the solar panels, and the spacecraft itself with the plasma exhaust. The electric propulsion transport module with the solar panels is jettisoned from the spacecraft before the arrival to the Jupiter system.

Braking propulsion system module provides corrections during the cruise, and the braking impulses near Jupiter and the forming the orbit around Europa. The propulsion system module consists of two propellant tanks, the main cruise engine, four thruster units; it includes also valves and constructional elements. Four identical spherical tanks with a capillary intake are used; two are intended for the oxidizer, and two for the fuel. The cruise propulsion system consists of four engines with the general traction of $\sim 1600 \mathrm{~N}$ and a specific impulse of $3000 \mathrm{~m} / \mathrm{s}$. The thruster units provide operating moments for attitude control and stabilization, and also velocity impulses for small trajectory corrections. Each block consists of four engines with traction of 50 and one engine with traction of 10 .

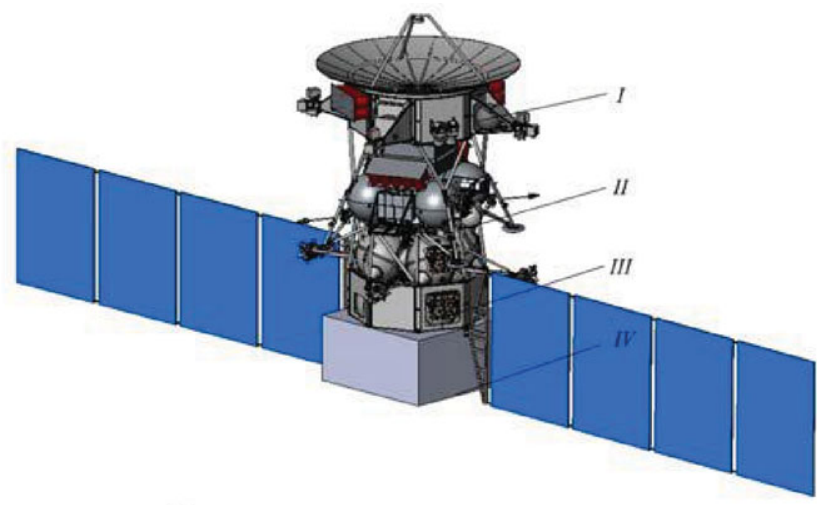

IV
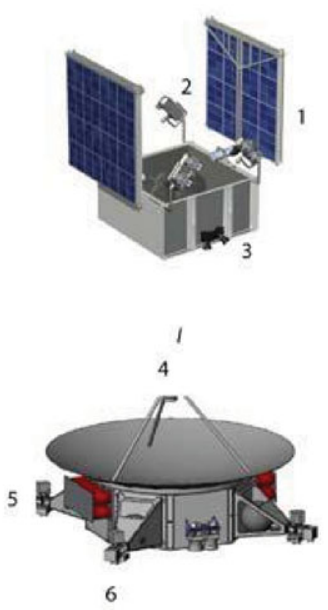

III

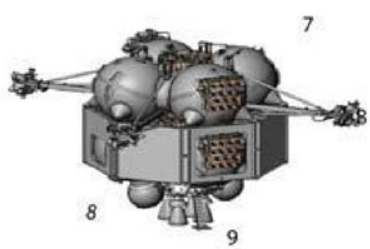

II

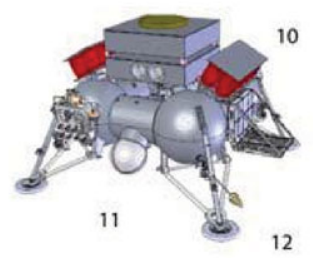

Figure 1. Europa Lander mission elements : I.- Orbiter: 4- High-gain antenna, 5- attitude control thrusters, 6- nuclear power units; II. - Landing module: 10 - nuclear power units, 11- science payload compartment; 12 - landing system; III. - Braking propulsion system: 7 propellant tanks, 8- structure, 9- main engine; IV.- Electroroket propulsion system: 1solar panels, 2- electrojet engines, 3 - attitude control thrusters. Xenon tanks are inside. 
Table 2. Estimated mass budget of the Europa mission employing electrorocket trust during interplanetary cruise. Upper stage Breeze booster is not included.

\begin{tabular}{lc}
\hline Unit & Mass, kg \\
\hline Orbital module & 395 \\
Landing module & 550 \\
Braking propulsion system module & 385 \\
Electrorocket propulsion system module & 860 \\
Intermediate structure & 70 \\
SC without propellant & $\mathbf{2 2 6 0}$ \\
EPS module propellant (xenon) & 1435 \\
Propulsion system module propellant & 2005 \\
Landing module propellant & 660 \\
SC with propellant & $\mathbf{6 3 6 0}$ \\
\hline
\end{tabular}

The orbital module is the principal structural element of the spacecraft. The systems of the orbital module provide the control at all stages of the flight, from cruise trough the insertion into the orbit around Europa. Redundant control system of the lander remains in reserve. This allows to increase the survivability of the spacecraft. The attitude control and stabilization of the orbiter is supported by reaction wheels, and a set of one-component chemical thrusters. The electric and thermal energy for the orbiter is provided by radioisotope thermoelectric generators (RITEG). Electric power generated by one standard RITEG is about $7 \mathrm{~W}$. To provide for necessary power of $210 \mathrm{~W}$, the RITEGs are integrated in two identical blocks (15 RITEGs in each).

During the cruise, maneuvers in the Jupiter system, and once inserted into the orbit around Europa the orbiter serves for science observations. After the lander is released, the main goal of the orbiter turns to relaying. The scientific instruments and service systems are located in the shielded compartment of the orbiter. The mass allocated for science instruments is $50 \mathrm{~kg}$. A high-gain 4-m antenna for communication with the Earth is fixed on the top of the instrument container.

The lander systems should provide $\Delta V$ to deorbit Europa, soft landing on the surface, and the realization of science program. The distinct feature of the Europa lander (w.r.t. Lunar landers) is the use of the monocomponent fuel on the base of hydrazine for the main brake engine. It allows minimizing the pollution of the surface. The lander includes a block of fuel tanks of the propulsion system, realized in the form of four spherical tanks connected by cylindrical spacers. The landing feet are fixed to each fuel tank.

Taking into consideration a high level of radiation, the scientific equipment and service systems are located in shielded instrument compartments with aluminum alloy walls of $>10 \mathrm{~mm}$ thickness. The mass allocated for science instruments and related subsystems (sampling systems, booms etc. is $\sim 50 \mathrm{~kg}$. Taking into account remoteness from the Sun, the lander like the orbiter uses RITEG as a source of electric and thermal energy. Data exchange with the Earth is provided through the orbiter.

The heritage from previous developments. The Europa's Lander mission will extensively use the heritage from already developed modules, units and systems which have passed a full cycle of ground-based and flight. The electrorocket transport module is being developed within the Russian Federal Space Program as a unified service framework. The first flight opportunity will be in the frame of Electro-M geostationary meteo-satellite (2014). It will also be used for Interheliozond mission for solar studies, also targeting the launch date in 2014.

Most of the heritage comes from developments within the frame of Phobos Sapmle Return Mission (Phobos-Soil) in preparation for the launch in 2011 (Zelenyi et al., 2010b). The orbital module and the propulsion system are already developed and are the part 
of Phobos-Soil. Recently they have passed a full cycle of qualification. The same type of orbital module will be used for the Luna-Globe (2013) project and is planned as a basis of Mars-Net (2016) and Venera-D (2018) orbiters.

Russia has a long experience in automated landing on the Moon and planets. A number of successful landings on the Moon have been realized from $70^{t h}$ to $80^{t h}$ years. Algorithms of landing are developed and will be renewed during another lunar mission Luna-Resource, which includes landing.

Nevertheless, a number of the critical technologies has to be developed at the earliest stages of the project. A particular attention will be paid to electronic components tolerant to extremely high radiation doze. Radioisotope thermoelectric generators which developed now in the frame of Luna-Resource program, have to be qualified for application on Europa. A lot of ground experiments is required to test the technology of low-temperature of ice drilling.

\section{References}

Abyzov, S. S. 1993, In Antarctic Microbiology (Ed. E. Friedmann), Wiley \& Sons, New York, USA, pp. $265-295$.

Anderson, J. D., Schubert, G., Jacobson, R. A., Lau, E. L., Moore, W. B., \& Sjogren, W. L. 1998, Science, 281, 2019-2022.

Balint, T. 2004, JPL report D30050, 60 p. NASA JPL/California Institute of Technology, Pasadena, CA, September 22

Billings, S. E. \& Kattenhorn, S. A. 2005, Icarus 177, 397-412

Blanc, M., Alibert, Y., Andre, N., et al. 2009, Experimental Astronomy, 23, 849-892

Castello, J. D. \& Rogers., S. O. 2005, Life in ancient ice, Princeton Univ. Press. 307 p

Chyba, C. \& Sagan, C. 1992, Nature, 355, 125-132

Chyba, C. F. 2000, Nature, 403, 381-382

Chyba, C. F. \& Phillips, C. B. 2002, Europa as an Abode of Life. Origins of Life and Evolution of the Biosphere, 32, 47-68

Clark, K., Greeley, R., Pappalardo, R., \& Jones, C. 2007, Europa Explorer Mission Study: Final Report, JPL report D41283.

Clark, K., Stankov, A., Pappalardo, R. T., Greeley, R., Blanc, M., Lebreton, J.-P., \& van Houten, T. 2009, The Europa Jupiter System mission, 40th Lunar and Planetary Science Conference, March 23-27, The Woodlands, Texas, id.2338

Cody, G. D. et al. 2000, Science, 289, 1337-1340.

Colburn, D. S. \& Reynolds, R. T. 1985, Icarus, 63, 39-44

Conrad, P. G. \& Nealson, K. H. 2001, Astrobiology, 1, 15-24

Cooper, J. F., Philips, C. B., Green, J. R., Wu, X., Carlson, R. W., Tamppari, L. K., Terrile, R. J., Johnson, R. E., Eraker, J. H., \& Markis, N. C. Europa exploration: science and mission priorities, The future of solar system exploration, 2003-2013, ASP conference series, v.272, M. V. Sykes, ed., 2002.

Figueredo, P. H. \& Greeley R. 2004, Icarus, 167, 287-312

Hand, K. P. \& Chyba, C. F. 2007, Icarus, 189, 424-438

Hoppa, G. V., Tufts, B. R., Greenberg, R., \& Geissler, P. E. 1999, Science, 285, 1899-1902

Hussmann, H., Spohn, T., \& Wieczerkowski, K. 2002, Icarus, 156 (1), 143-151.

Gershman, R. 1999, Conceptual design of a Europa Lander mission, IEEE Aerospace conference, v.1, pp. $421-431$

Gershman, R., Nilsen, E., \& Oberto, R. 2003, Acta Astronautica, 52, 253-258

Gilichinsky, D. A., Wilson, G. S., Friedmann, E. I., et al. 2007, Astrobiology, 7, 275-311

Gowen, R. A., Smith, A., Fortes, A. D., et al. 2010, Adv. Space Res., in press.

Greeley, R., Sullivan, R., Klemaszewski, J. et al. 1998a, Icarus, 135, 4-24

Greeley, R., Sullivan, R., Coon, M. D., Geissler, P. E., Tufts, B. R., Head, J. W., Pappalardo, R. T., \& Moore, J. M. 1998b, Icarus, 135, 25-40 
Greeley, R. \& Johnson, T. 2004, Report of the NASA Science Definition Team for the Jupiter Icy Moons (JIMO), Technical Report, National Aeronautics and Space Administration, February 13

Greenberg, R., Hoppa, G. V., Tufts, B. R., Geissler, P., Riley, J., \& Kadel, S. 1999, Icarus, 141, 263-286

Greenberg, R., Leake, M. A., Hoppa, G. V., \& Tufts, B. R. 2003, Icarus, 161(1), 102-126

Irwin, L. N. \&. Schulze-Makuch, D. 2003, Astrobiology, 3, 813-820

Johnson, T. V. \& McCord, T. B. 1971, Astroph. J., 169, 589

Kargel, J. S., Kaye, J., Head, J. W. III, Marion, G. M., Sassen, R., Crowley, J., Prieto, O., Grant, S. A., \& Hogenboom, D. 2000, Icarus, 148, 226-265

Kivelson, M. G., Khurana, K. K., Russell, C. T., Volwerk, M., Walker, R. J., \& Zimmer, C. 2000, Science, 289, Is. 5483, 1340-1343

Korablev, O., Gerasimov, M., Dalton, J. B., Lebreton, J.-P., Hand, K., \& Webster, C. 2010, Submitted to Ads. Space Res.

Kuiper, G. P. 1957, Astron. J., 62, 295-295

Kuskov, O. L. \& Kronrod, V. A. 2005, Icarus, 177, 550-569

Langmaier, J. \& Elliott J. 2008, JPL publication 08-1, January

Leith, A. \& McKinnon, W. 1996, Icarus, 120, 387-398

Marion, G. M., Fritsen, C. H., et al. 2003, Astrobiology, 3, 785-811

Matousek, S. 2007, Acta Astronautica, 61, 932-939

McCord T. B., G. B. Hansen, F. P. Fanale, et al. 1998, Science, 280, 1242-1245

McEwen, A. S. 1986, Nature, 321, 49-51

Mileikowsky, C., Cucinotta, F. A., Wilson, J. W., et al. 2000, Planet. Space Sci., 48, 1107-1115

Moroz, V. I. 1965, Astronomicheskii Zhurnal, 42, 1287

Mukhin, L. M. 1976, Origins of Life 7, 355-368

Mukhin, L. M., Gerasimov, M. V., \& Safonova, E. N. 1989, Nature, 340, 46-48

Nansen, O. L. 1975, Icarus, 26, 24-29

Ojakangas, G. \& Stevenson, D. 1989, Icarus, 81, 242-270

Pappalardo, R. T., Belton, M. J. S., Breneman, H. H., et al. 1999, J. Geophys. Res., 104, E10, $24015-24056$

Pappalardo, R. T., Clark, K., Greeley, R., et al. 2007, Europa Explorer: A Mission to Explore Europa and Investigate Its Habitability, DPS meeting \#39, \#28.02; Bull. American Astronomical Soc., 39, 465

Phillips, C. B., McEwen, A. S., Hoppa, G. V. et al. 2000, J. Geophys. Res., 105, 22579-22598

Pierazzo, E., Chyba \& C. F. 2002, Icarus, 157, 120-127

Pilcher, C. B., Ridgway, S. T., \& McCord, T. B. 1972, Science, 178, 1087-1089

Podzolko, M. V., Getselev, I. V., Gubar', Yu. I., \& Veselovsky, I. S. 2009, Solar System Research, $43,116-120$

Pollack, J. B., Witteborn, F. C., Edwin, F. E., Strecker, D. W., Baldwin, J. B., \& Bunch, B. E. 1978, Icarus, 36, 271-303

Prieto-Ballesteros, O., F. Gomez et al. 2010, Adv. Space Res., submitted

Priscu, J. C. Perennially ice covered Antarctic lakes: oases for life and models for other icy worlds. Astrobiology in Russia workshop, March 25-29, 2002, St. Petersburg, Russia, p. $116-130$

Priscu, J. C., Adams, E. E., Lyons, W. B., Voytek, M. A., Mogk, D. W., Brown, R. L., McKay, C. P., Takacs, C. D., Welch, K. A., Wolf, C. F., Kirshtein, J. D., \& Avci, R. 1999, Science, $286,2141-2144$

Reynolds, R. T., Squyres, S. W., Colburn, D. S., \& McKay, C. P. 1983, Icarus, 56, 246-254

Russell, C. 2003, The Cassini-Huygens Mission, Kluwer

Sassen, R., S. Joye, S. T. Sweet, D. A. DeFreitas, A. V. Milkov, \& I. R. MacDonald 1999, Organic Geochem., 30, 485-497

Schenk, P. M. 2002, Nature, 417, 419-421

Schenk, P. M., McKinnon W. B. 1989, Icarus, 79, 75-100

Schenk, P., Matsuyama, I., \& Nimmo, F. 2008, Nature, 453, 368-371 
Schilling, N., Khurana, K., \& Kivelson, M. G. 2004, J. Geophys. Res. 109, doi:10.1029/2003JE002166

Sieger, M. T., Simpson, W. C., \& Orlando, T. M. 1998, Nature, 394, 554-556

Showman, A., \& Han, L. 2004, J. Geophys. Res. 109 (E1), CiteID E01010

Stevenson, D. J. 2000, Science, 289, 1305-1307

Tobie, G., Choblet, G., \& Sotin, C. 2003, J. Geophys. Res. 108 (E11), 10-1, doi:10.1029/2003JE002099

Vorobyova E., Soina V., Gorlenko M., et al. 1997, FEMS Microbiol. Rev., 20, 277-290

Vorobyova, E. A., Soina, V. S., \& Mulyukin, A. L. 1996, Adv.Space.Res., 15, 103-108

Williams, K. K., \& Greeley, R. 1998, Geophysical Res. Lett., 25, 4273-4276

Whitman, W. B., Coleman, D. C., \& Wiebe, W. J. 1998, Proc. Natl. Acad. Sci. U.S.A., 95, $6578-6583$

Zahnle, K., Schenk, P., Levison, H., \& Dones, L. 2003, Icarus, 163(2), 263-289

Zahnle, K., Alvarellos, J. L., Dobrovolskis, A., \& Hamill, P. 2008, Icarus, 194(2), 660-674

Zelenyi, L. M., Zakharov, A. V., Polischuk, G. M., \& Martynov, M. B. 2010a, Solar System Res., $44,15-25$

Zelenyi, L., Korablev, O., Martynov, M., et al. 2010b, Submitted to Adv. Space Res.

Zimmer, C., Khurana, K. K., \& Kivelson, M. G. 2000, Icarus, 147(2), 329-347

Zimmerman W. F., James Shirley, Robert Carlson, Tom Rivellini, Mike Evans, Europa Small Lander Design Concepts, American Geophysical Union, Fall Meeting 2005, abstract \#P54A-08, 2005. 